\title{
Structural investigations of copper nanorods by high-resolution TEM
}

\author{
I. Lisiecki and A. Filankembo \\ Université Paris VI, U.P.M.C. Laboratoire SRSI, Boite Postal 52, 4 Place Jussieu, F-75005 Paris, France \\ and CEA-Saclay, DSM-DRECAN-SCM, F-91191, Gif-sur-Yvette, France \\ H. Sack-Kongehl and K. Weiss \\ Fritz-Haber-Institut der MPG, Abteilung AC, Faradayweg 4-6, D14195 Berlin, Germany \\ M.-P. Pileni \\ Université Paris VI, U.P.M.C. Laboratoire SRSI, Boite Postal 52, 4 Place Jussieu, F-75005 Paris, France \\ and CEA-Saclay, DSM-DRECAN-SCM, F-91191, Gif-sur-Yvette, France
}

J. Urban*

Fritz-Haber-Institut der MPG, Abteilung AC, Faradayweg 4-6, D14195 Berlin, Germany

(Received 1 March 1999; revised manuscript received 22 July 1999)

\begin{abstract}
Well-defined long $\mathrm{Cu}$ rods having a length of the order of $1 \mu \mathrm{m}$ and diameters of several nanometers were prepared by reduction of copper compounds. After deposition on amorphous carbon films, high-resolution transmission electron microscopy and electron diffraction were performed in order to explain the structure of the rods. By applying computer simulations with multislice calculations, the particle structure was obtained. The rods were held to be truncated decahedra with a fivefold symmetry. It could be shown that most particles were oriented in the [001] direction with respect to the substrate for one of the five deformed tetrahedral subunits, i.e., the fivefold axis very often was parallel to the surface of the substrate. It was also proven that the $\mathrm{Cu}$ fcc bulk structure containing stacking faults had to be excluded as a possible structural model. Also, truncated icosahedral structures or icosahedra with additional intermediate planes did not serve to explain the experimental process. Icosahedra are often observed together with decahedral structures for particles with a spherical-like morphology. Due to the presence of surfactant, only growth in the direction of the fivefold axis of decahedra was possible, resulting in long needlelike rods.
\end{abstract}

\section{INTRODUCTION}

During the last few years extensive efforts had been undertaken to control the size and shape of nanocrystals. ${ }^{1}$ Metal nanoparticles $^{1,2}$ are of great advantage because of their electronic properties ${ }^{3-6}$ compared to the bulk phase, additionally they very often act as active catalysts. ${ }^{7}$ Colloids can be used as templates ${ }^{8}$ to control the size $\mathrm{e}^{3,4,6,9}$ and shape $\mathrm{e}^{1,4,9-12}$ of particles. In terms of the growth of particles, some analogies between surfactant self-assemblies and natural media have been proposed. ${ }^{1}$ In both cases, the growth of particles needs a supersaturated medium where the nucleation takes place. Increasingly, chemists are contributing to the synthesis of advanced materials with enhanced properties by using colloidal assemblies as templates. In previous papers, it was shown that oil in water micelles can be used to control the size of spherical copper metal particles. ${ }^{3,4,6}$ Recently, it was demonstrated that the shape of copper metal particles strongly depends on the colloidal structure in which the chemical reduction of copper (II) bis (2-ethylhexylsulfosiccinate) $\left[\mathrm{Cu}(\mathrm{AOT})_{2}\right]$ takes place. ${ }^{9-12}$ When the template is made of interconnected cylinders, long rods of copper are obtained. Slight changes in the experimental conditions induce distinct modifications of the shape and size of the rods. ${ }^{10-12}$ Unfortunately, these studies lack in not having been structurally studied. In the present paper, we report, information on the structure of such rods. The technique of high-resolution transmission electron microscopy (HRTEM), together with electron diffraction, is one of the most important techniques employed in the structural determination of small nanoparticles (clusters) in the size range of $1-10 \mathrm{~nm} .{ }^{13,14}$ In order to study the reactivity of such particles, data of the structure in combination with possible catalytic activities together with defined size distribution are of great importance.

\section{SYNTHESIS OF COPPER METAL RODS}

The copper metal particles are prepared by reduction of $\mathrm{Cu}(\mathrm{AOT})_{2}$, in $\mathrm{Cu}(\mathrm{AOT})_{2}$-isooctane-NaCl-water colloidal self-assemblies. Hydrazine is used as the reducing agent, with an overall concentration of $0.15 \mathrm{M}$ and the reaction takes place under a $\mathrm{N}_{2}$ atmosphere starting immediately after hydrazine has been added to the colloidal solution. In a pyrex tube, $3 \mathrm{ml}$ of $5 \times 10^{-2} \mathrm{M} \mathrm{Cu}(\mathrm{AOT})_{2}$-isooctane are mixed with $36.1 \mu \mathrm{l}$ of $10^{-1} \mathrm{M}$ sodium chloride previously dissolved in water. The $\mathrm{Cu}(\mathrm{AOT})_{2}$, water, and chloride concentrations are $5 \times 10^{-2}, 0.55$, and $10^{-3} \mathrm{~mol} \mathrm{dm}^{-3}$, respectively. The system is kept for $20 \mathrm{~h}$ at room temperature (20$22^{\circ} \mathrm{C}$ ). Then, $21.9 \mu \mathrm{l}$ of $20.6 \mathrm{~mol} \mathrm{dm}^{-3}$ hydrazine is added to the colloidal solution which is vigorously stirred. Under these conditions, the ratio of $\mathrm{Cu}(\mathrm{AOT})_{2}$ to hydrazine is equal to $1 / 3$. The colloidal assembly immediately turns dark, which is due to the reduction of $\mathrm{Cu}(\mathrm{AOT})_{2}$ to $\mathrm{Cu}(\mathrm{O})$ and the solution becomes darker with time. After $2 \mathrm{~h}$, a drop of the so- 


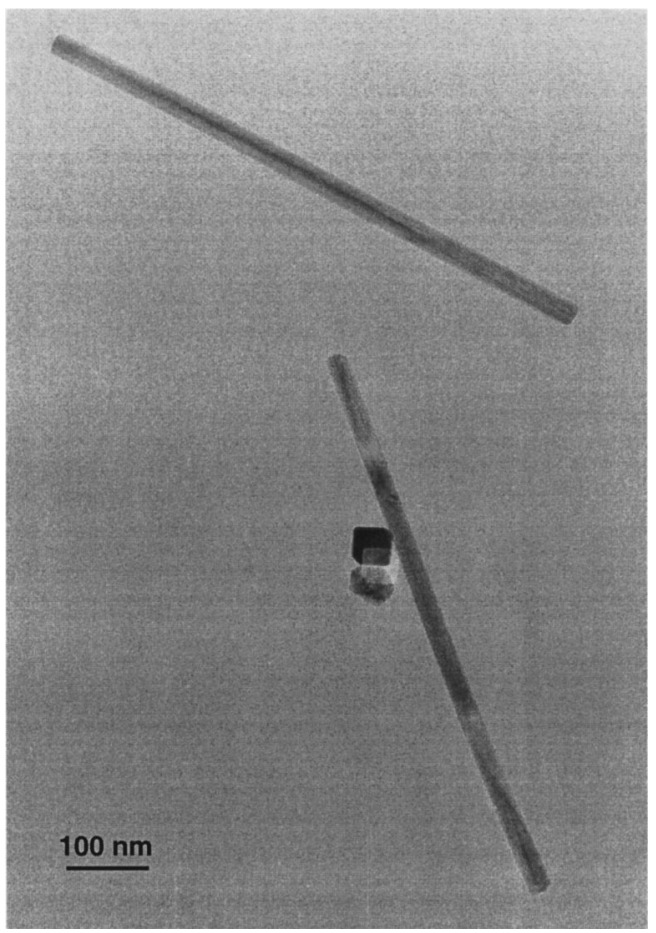

FIG. 1. Overview electron micrograph $\mathrm{Cu}$ rods with an electron optical magnification of $57000 \times$.

lution is placed on a carbon film supported by a copper grid and examined by TEM. Investigations which were performed a few minutes after the hydrazine was added to the colloidal system shared the presence of water in oil interconnected cylinders. ${ }^{12}$ In terms of the local $\mathrm{Cu}(\mathrm{AOT})_{2}$ number of molecules at the interface, this turns out to be much higher than the overall concentration given above. Thus, when the chemical reduction starts, the space is limited by the waterin-oil cylinders in a very high supersaturation regime. The copper nuclei formed are close to the interface, which favors crystallization in a preferential direction. With time, the production of copper nuclei is reduced and the viscosity at the interface remains still high. Finally, slow crystallization, which allows the controlling of the physical parameters, also allows the formation of long rods of copper.

\section{HRTEM OF Cu RODS}

In order to study the structure of the $\mathrm{Cu}$ rods, HRTEM was performed. The images were obtained with a Philips CM200 FEG microscope operating at $200 \mathrm{kV}, C_{s}$ $=1.35 \mathrm{~mm}$, with an information limit better than $0.18 \mathrm{~nm}$. Together with the high-resolution images, electron diffraction was also performed and, finally, after digitizing the images with a pixel size of about $0.03 \mathrm{~nm}$, the power spectra (PS), i.e., the square of the Fourier-transform of the image, were calculated.

To demonstrate the successful preparation of long thin rods having a length of the order of $1 \mu \mathrm{m}$ and diameters of about $25 \mathrm{~nm}$, overview electron micrographs of low magnification were taken. In Fig. 1 such an overview image is shown. The total image size corresponds to 800 $\times 1150 \mathrm{~nm}^{2}$.

With respect to larger magnification, i.e., images with

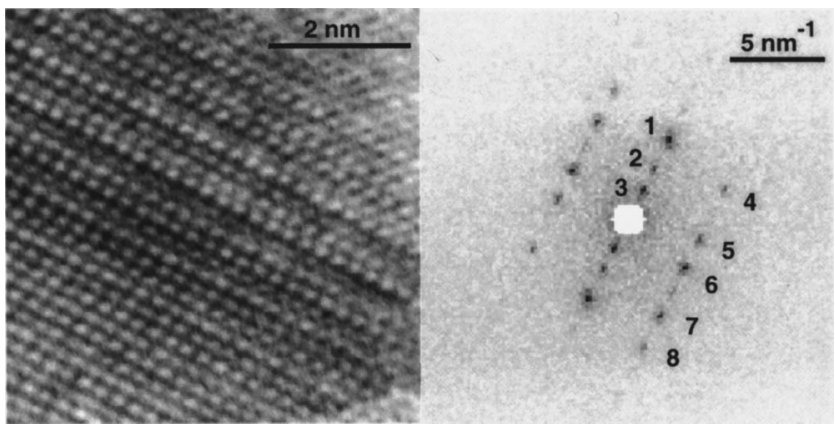

FIG. 2. Detail of a copper rod. The right image represents the PS. The reflection spots are labeled from 1 to 8 with lattice parameters: $d_{1}=0.211 \mathrm{~nm}, \quad d_{2}=0.332 \mathrm{~nm}, \quad d_{3}=0.566 \mathrm{~nm}, \quad d_{4}=d_{7}$ $=0.184 \mathrm{~nm}, d_{5}=d_{6}=0.252 \mathrm{~nm}$, and $d_{8}=0.148 \mathrm{~nm}$. The angle between planes represented by reflections 5 and 6 is $27^{\circ}$.

atomic resolution, it was observed that many rods showed almost the same orientation. An example of a particle showing such a typical orientation is presented in Fig. 2. Here only a small part of the center of the rod is displayed together with the calculated PS, from which the following data could be obtained.

Three pairs of equatorial reflections marked as 1,2 , and 3 are observed together with sideband reflections at the top and the bottom of the PS marked as 4, 5, 6, 7, and 8. These reflections correspond to the lattice parameters: $d_{1}$ $=0.211 \mathrm{~nm}, \quad d_{2}=0.332 \mathrm{~nm}, \quad d_{3}=0.566 \mathrm{~nm}, \quad d_{4}=d_{7}$ $=0.184 \mathrm{~nm}, d_{5}=d_{6}=0.252 \mathrm{~nm}$, and $d_{8}=0.148 \mathrm{~nm}$. The accuracy is of the order of $\pm 2 \%$.

Reflections 1,4 , and 7 resemble data very close to the $\mathrm{Cu}(111)$, (200), and (020) plane spacings, respectively $(0.2087,0.1808$, and $0.1808 \mathrm{~nm})$. The angle between (200) and $(020)$ is $87^{\circ}$. The small deviations can be attributed to deformations within the particle. The appearance of the (200) and (020) reflections together with the (111) reflection proves that the particle must consist of more than one subunit (twinning). This finding will be discussed in below (structure of the particles). Reflections 5 and 6 represent planes with an angle of $27^{\circ}$ with respect to each other. The data of these and the other reflections will be explained in detail further below.

\section{TILT SERIES OF HRTEM IMAGES}

In order to obtain more information on structural details we also performed tilt series of the rods. The long axis of the rods was always taken as the tilt axis. The tilt angle with respect to the substrate of the above shown particle, cf. Fig. 2 , was defined as $0^{\circ}$. Surprisingly, after tilting the particles, a periodic sequence could be observed, i.e., every $36^{\circ}$ a complete repetition of the image, clearly displayed in the PS, was achieved, [cf. Figs. 3(c) and 5(c)]. A second different configuration by tilting around + and $-18^{\circ}$ was also observed. The reflections obtained under $\pm 18^{\circ}$ tilt angles; cf. Figs. 4(c) and 6(c) are labeled 1,2, and 3. Here again, the data obtained as $0.206,0.207$, and $0.179 \mathrm{~nm}$ closely resemble the (111) and (200) reflections of $\mathrm{Cu}$, respectively. The angle between planes which are represented by reflections 1 and 2 is $71.5^{\circ}$. The PS of Figs. 4(c) and 6(c), however, resemble that of a [110] zone axis image of the fcc structure although the peri- 

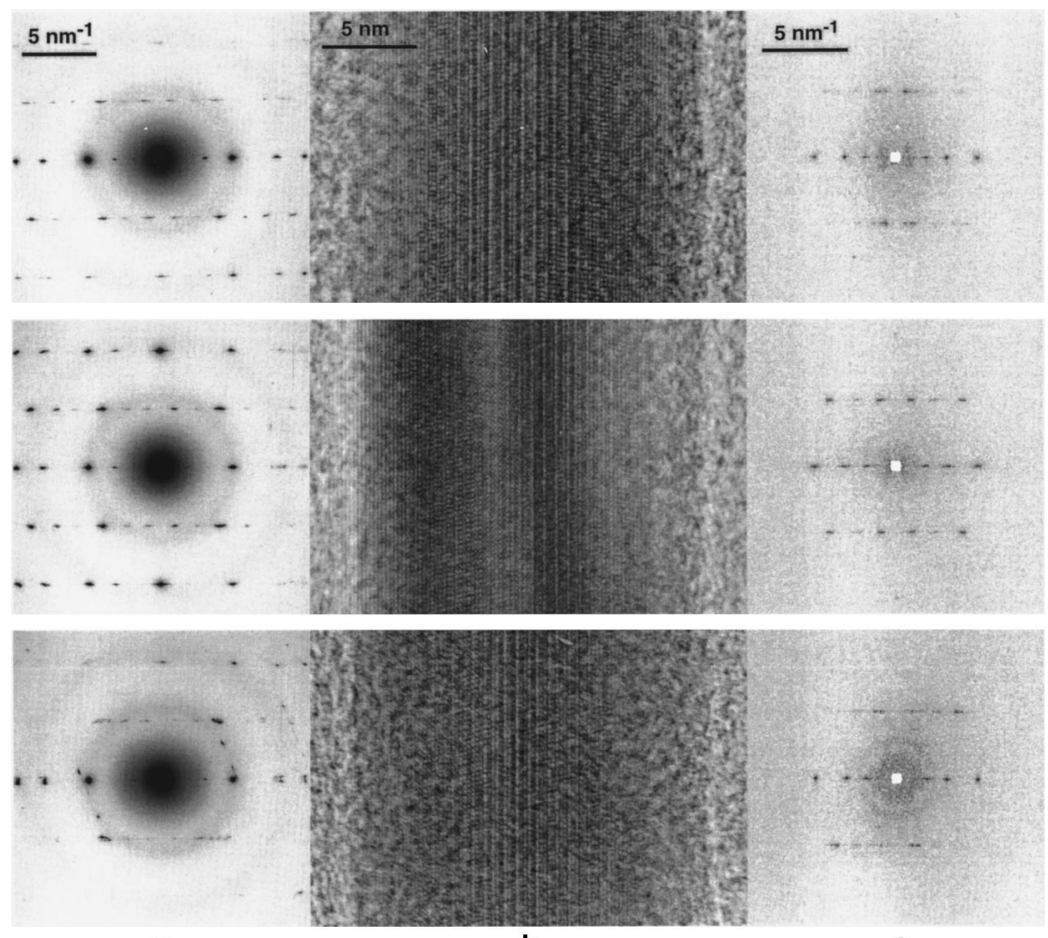

a

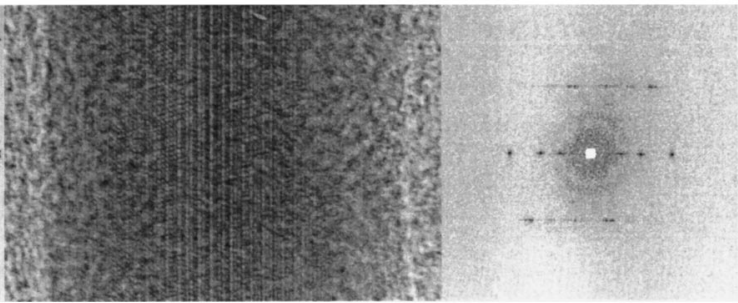

b

odic repetition for fcc structures as observed in the tilt series does not exist. Again it is held that the deviations are due to distortions in the rods. This conclusion is also strongly supported by the splitting of most reflections in the PS. Two typical examples of tilt series by $0^{\circ}, \pm 36^{\circ}$, and $\pm 18^{\circ}$ are shown in Figs. 3 and 4 and 5 and 6. In section (a) of the figures the electron diffraction is shown, (b) displays the real image, and (c) shows the calculated PS. From the real image it can be seen that for tilt angles $0^{\circ}$ and $\pm 36^{\circ}$ the central parts of the images show atomic resolution with strong contrast. The particles tilted by $\pm 18^{\circ}$, however, show a different behavior. Here, only on the left or right part can lattice planes be visualized, respectively. An interpretation thereof will be given below.

\section{STRUCTURE OF THE PARTICLES}

\section{A. fec with stacking faults}

The appearance of superstructure lines along the axis of the particles suggested an fcc structure with stacking faults along [111]. ${ }^{15,16}$ However, model calculations showed that the typical reflections labeled 5 and 6 in Fig. 2, with $27^{\circ}$ angles of the corresponding net planes, could never be explained by the fcc structure whatever the stacking. The same applied to the reflections labeled 1, 4, and 7. Such structure could also not explain the periodic appearance of the diffraction spots or the reflections in the PS after tilting around the long axis by $\pm 36^{\circ}$ or $\pm 18^{\circ}$, respectively.

\section{B. Decahedral and icosahedral structure}

It is well known ${ }^{17-19}$ that small $\mathrm{Cu}$ clusters, smaller than about $5 \mathrm{~nm}$ diameter, show the structure of decahedra or icosahedra, i.e., particles with fivefold symmetry, when prepared by the inert gas aggregation technique. ${ }^{20,21}$ This finding was also supported by the theoretical approaches of Refs. 24 and 25, and results on electron diffraction of Ref. 26. Decahedral particles consist of five deformed tetrahedral subunits. The common edge of the five subunits represents the fivefold axis which is larger by $5 \%$ than the other edges.

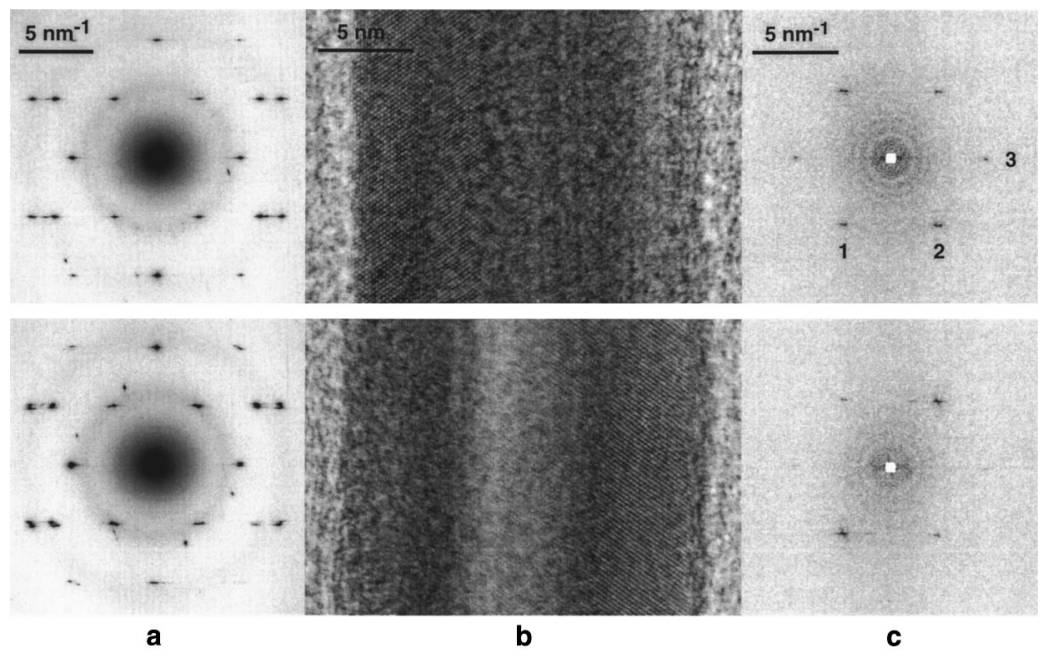

FIG. 4. Tilt series of sample 1: From top to bottom: $+18^{\circ}$ and $-18^{\circ}$, (a) Electron diffraction. (b) HRTEM image. (c) PS. Three pairs of reflections in the PS are labeled by 1,2 , and $3 . d_{1}$ $=0.206 \mathrm{~nm}, d_{2}=0.207 \mathrm{~nm}$, and $d_{3}=0.179 \mathrm{~nm}$. The angle between $d_{1}$ and $d_{2}$ is $71.5^{\circ}$. 

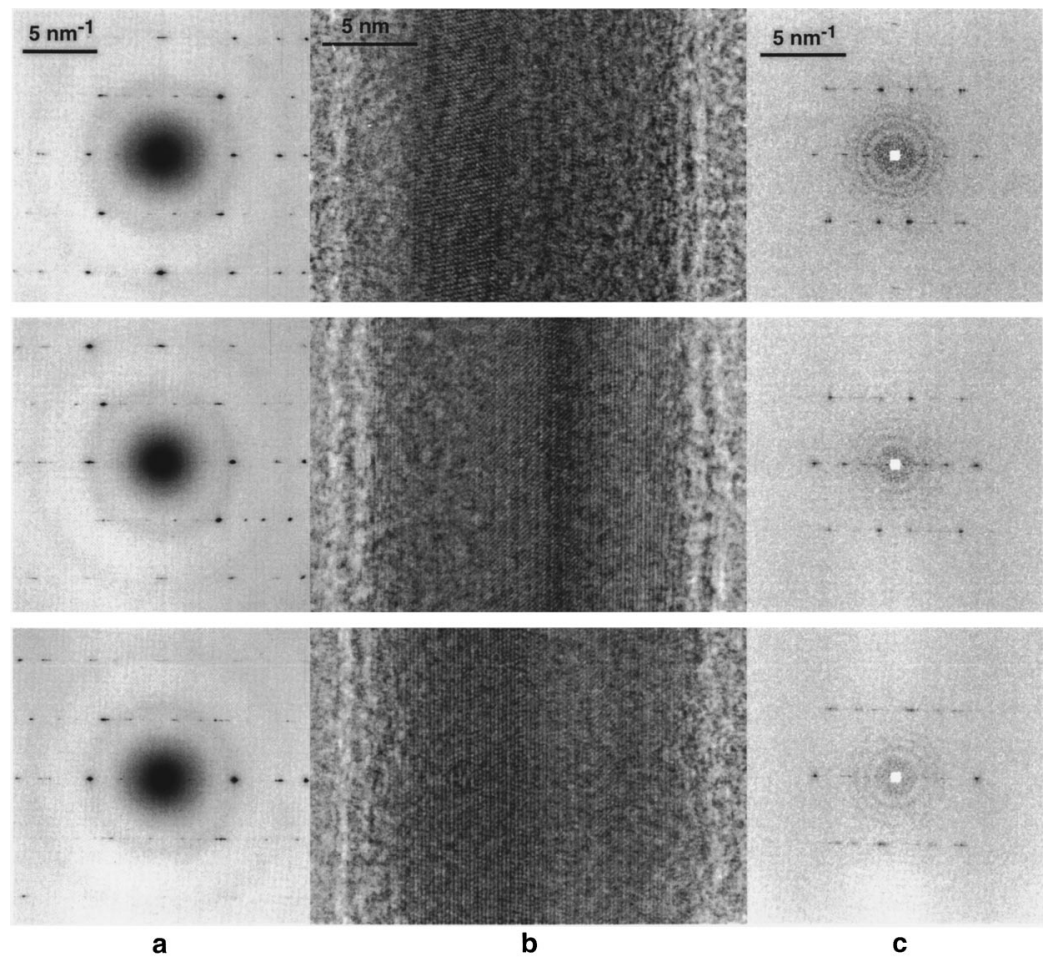

FIG. 5. Tilt series of sample 2: From top to bottom: $+36^{\circ}, 0^{\circ}$, and $-36^{\circ}$. (a) Electron diffraction. (b) HRTEM image. (c) PS.
Icosahedral particles consist of 20 deformed tetrahedral subunits. The deformation must be introduced in order to avoid gaps between the faces of adjoining subunits. The structure of such multiply twinned particles was discussed in detail elsewhere. ${ }^{27}$ For the present preparation, a structure with fivefold symmetry-but now as large truncated decahedra, or, in other words, decahedral particles with additional intermediate (110) planes-was introduced. The orientation of the particles with respect to the substrate was assumed to be [001] for one of the five deformed tetrahedral subunits which was previously defined as tilt angle $0^{\circ}$. Figures 7 (a) and 7(b) show decahedral models viewed along the fivefold axis which is defined in analogy to the fcc structure as the [110] axis. This axis is common for all five subunits. The subunits are labeled $T 1, \ldots, T 5$. Each consists of four (111) surfaces, i.e., (111), (-1-11), (1-1-1), and (-11-1). The (1-1-1) and (-11-1) surfaces are common faces for neighboring subunits, and the angle between the two planes is $72^{\circ}$ instead of $70.5^{\circ}$ for the fcc tetrahedral subunit. However, the angle between (111) and (-1-11) surfaces, i.e., top and bottom planes, is about $75^{\circ}$. It should also be mentioned that due to the deformation of the subunits, the lattice spacings for the (111) and (-1-11) spacings, which are equal, are slightly different from the (1-1-1) and (-11-1) spacings, which are also equal. In Figs. 7(a) and 7(b) the directions of the electron beams are $0^{\circ}$, $\pm 36^{\circ}$, and $\pm 18^{\circ}$, respectively. From this model it is obvious that viewing perpendicularly to (001), as indicated by arrow $0^{\circ}$ in the figure, a tilt by $\pm 36^{\circ}$, as shown by the arrows, always gives the same image. Furthermore, by tilting around $\pm 18^{\circ}$, another differently structured pattern is explained. It is also obvious from the model in Fig. 7(b) that for different tilts lattice planes can be imaged only in the outer right or left parts. For $0^{\circ}$ viewing, from $T 3$ (-11-1) planes are imaged, and from $T 4$ (1-1-1) planes are imaged. For $+36^{\circ}$ viewing, (-11-1) planes of $T 5$ and (1-1-1) plane of $T 1$ are imaged. In the case of $-36^{\circ}$ tilts from $T 1$ and $T 2$,
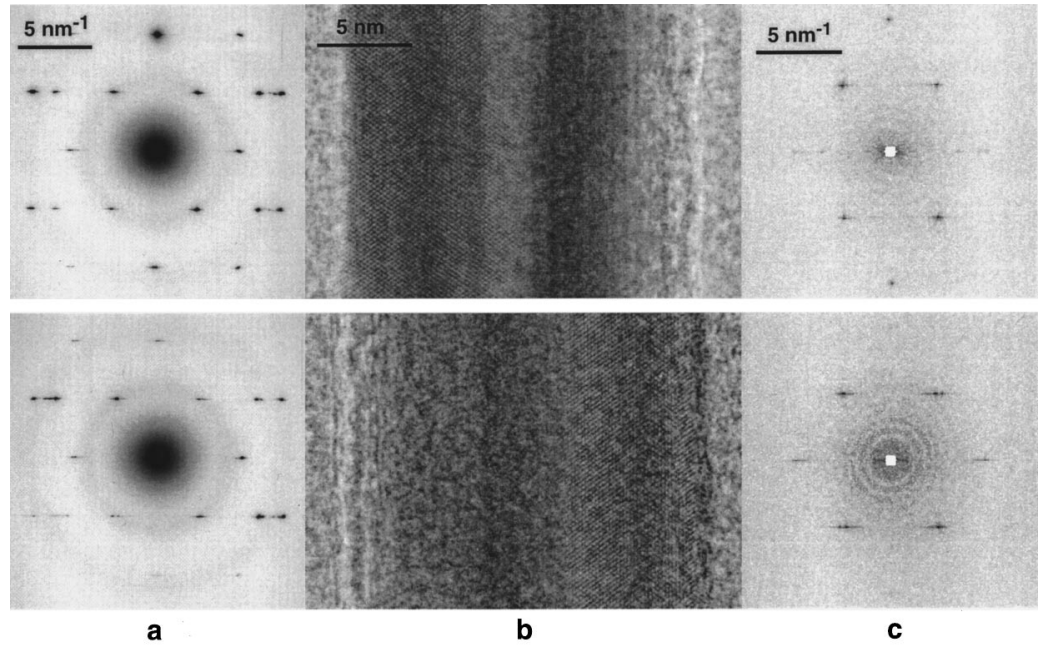

FIG. 6. Tilt series of sample 2: From top to bottom: $+18^{\circ}$ and $-18^{\circ}$. (a) Electron diffraction. (b) HRTEM image. (c) PS. 

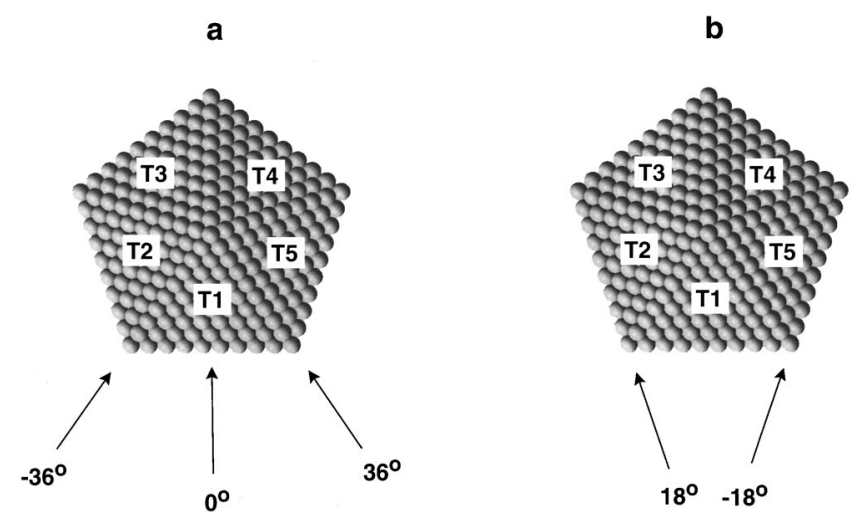

FIG. 7. Decahedral model particle viewed along the fivefold axis. The deformed subunits are labeled $T 1, \ldots, T 5$. The arrows indicate the directions of the electron beam by tilting the model: (a) $0^{\circ}$ and $\pm 36^{\circ}$. (b) $\pm 18^{\circ}$.

(1-1-1) and (-11-1) planes are imaged, respectively. The other reflections in the PS and diffraction images will be explained by means of computer simulations below. From Fig. $7\left(\right.$ b) it can be concluded that under $+18^{\circ}$ the $(001)$ planes of $T 2$, which are parallel to the beam, are imaged on the left-hand side only, while this occurs for $-18^{\circ}$ on the right-hand side. For the latter case, the (001) planes of $T 5$ are imaged.

By using the multislice technique, ${ }^{22}$ computer simulations for truncated decahedral particles consisting of 8224 atoms were performed. The truncation is such that eight intermediate (110) planes are obtained. The particle has a diameter of $5.9 \mathrm{~nm}$ and a length of $6.2 \mathrm{~nm}$. The calculation was performed for the above-discussed typical tilt angles of $0^{\circ}$, $\pm 18^{\circ}$, and $\pm 36^{\circ}$. The corresponding PS were also calculated. As expected, the periodic repetitions could also be achieved by the model calculation. Figure 8 shows models, computer simulations, and PS for such truncated decahedra viewed along the fivefold axis ([110] direction), in a [001] direction, and parallel to a (001) plane ([1-10] direction), i.e., tilt angles $0^{\circ}$ and $18^{\circ}$, respectively, for the latter two orientations. Obviously, a long rod would not be expected to be oriented along the fivefold axis with respect to the substrate. This orientation was not observed in the case of the presently prepared rods, although this is often observed for spherical particles, ${ }^{23}$ which will not be discussed here. For both tilts, all reflections visible in the PS of the experimental image are also obtained in the PS of the computer simulations.

The diffraction images shown in Figs. 3-6 show more reflections than given in the PS of the images and the computer simulations. We therefore show magnified images of the diffraction images of Figs. 3 and 4 for $0^{\circ}$ and $18^{\circ}$ tilts in Figs. 9(a) and 9(b), respectively. Most of the reflections are labeled by their corresponding Miller indices. In both cases different directions corresponding to different subunits are observable. For each direction a corresponding lattice is drawn in the images: one with dashed lines with the indices given in italic letters, and the other with solid lines with indices in bold letters. There are many reflections visible which do not lie on the lattices. They correspond to multiple diffraction spots whose origins will be discussed for some examples.
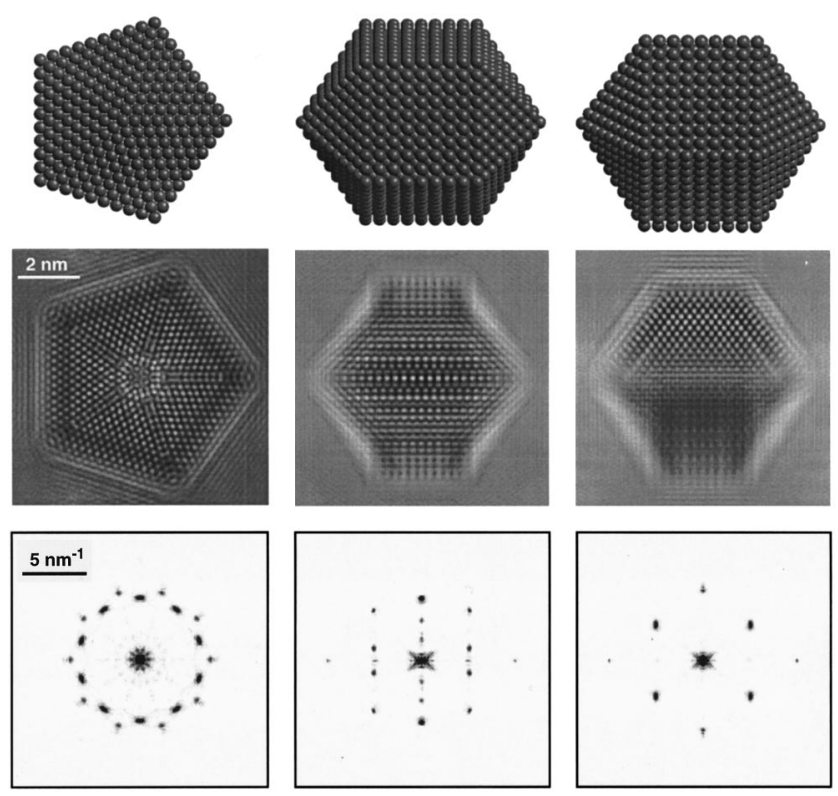

FIG. 8. Models, calculated images and PS for truncated decahedral particles for three different orientations. From left to right: fivefold axis, in [001] direction and in a [1-10] directions [parallel to a (001) plane], i.e., $0^{\circ}$ and $18^{\circ}$, perpendicular to the fivefold axis for the latter two.

$0^{\circ}$ tilt: Reflections 1, 4, and 7 labeled in Fig. 2 belong to the (111) and (200) families. Reflection 1 is produced by subunits $T 3$ and $T 4$, cf. Fig. 7(a). However, $T 3$ produces a (1-11) reflection while $T 4$ is responsible for a (1-1-1) reflection, these reflections are equal in spacing and position in reciprocal space, as can be seen from the diffraction patterns in Fig. 9(a). Reflections 4 and 7 are the (020) and (200) reflections, respectively, which are scattered from $T 1$. There are also two weaker reflections visible in Fig. 8, i.e., (110) and (220). They are created by all subunits $T 1-T 5$. However, from the diffraction patterns in Fig. 9(a) the (110) reflection is not visible. This reflection is also not imaged in Fig. 2. In real fcc structures this reflection is forbidden and appears to be very weak in the model calculation, seeing as there is a small deviation from the fcc structure.

In the diffraction image of Fig. 9(a) the orientations of the scattering subunits are the [-112], [1-12], and [001] directions for fcc structures, although deviations from this structure exist. This interpretation is only given in analogy to the fcc structure seeing that the decahedral structure is not a crystallographic symmetry. The dashed lines belong to the [-112] orientation of $T 3$ and the [1-12] orientation of $T 4$. The full lines are from the [001] oriented subunit $T 1$. Indices of the reflections are given in the figure. The angle between (020) and (200) [cf. Fig. 9(a)] is slightly smaller than $90^{\circ}$ (cf. the angle between $d_{4}$ and $d_{7}$ in Fig. 2 is $87^{\circ}$ ), which again is due to the deformation of the tetrahedral subunits for the fcc structure in order to create the decahedral structure.

$\pm 36^{\circ}$ tilt: By this tilt the reflection patterns are the same as for $0^{\circ}$. However, the reflections there are caused by different subunits. For the (111) family, $T 1$ and $T 5$ for $+36^{\circ}$ and $T 1$ and $T 2$ for $-36^{\circ}$. For the (200) family, $T 3$ for $+36^{\circ}$ and $T 4$ for $-36^{\circ}$. The (220) reflection is again due to all five subunits. In the diffraction image in Fig. 9(a), the reflections are again obtained by the scattering of different subunits in 


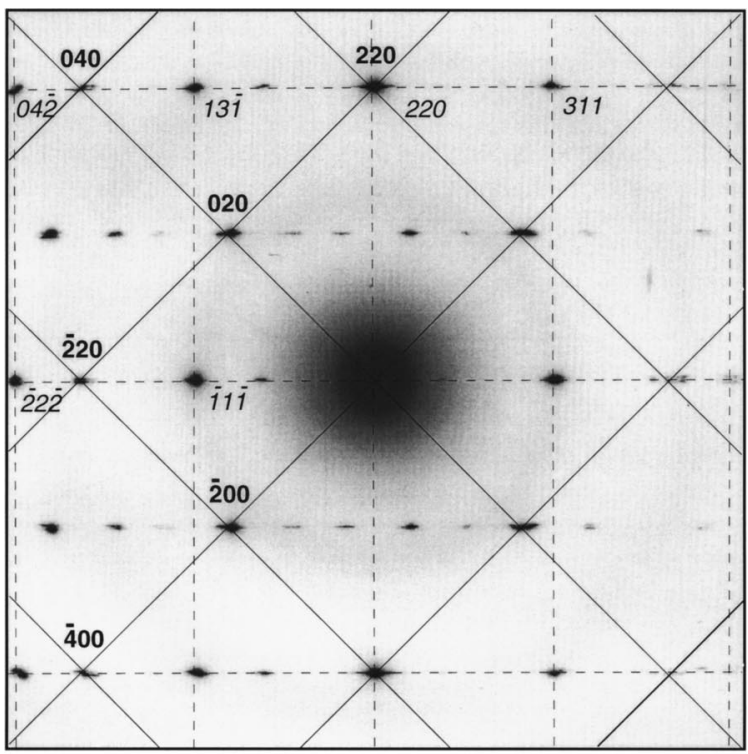

(a)

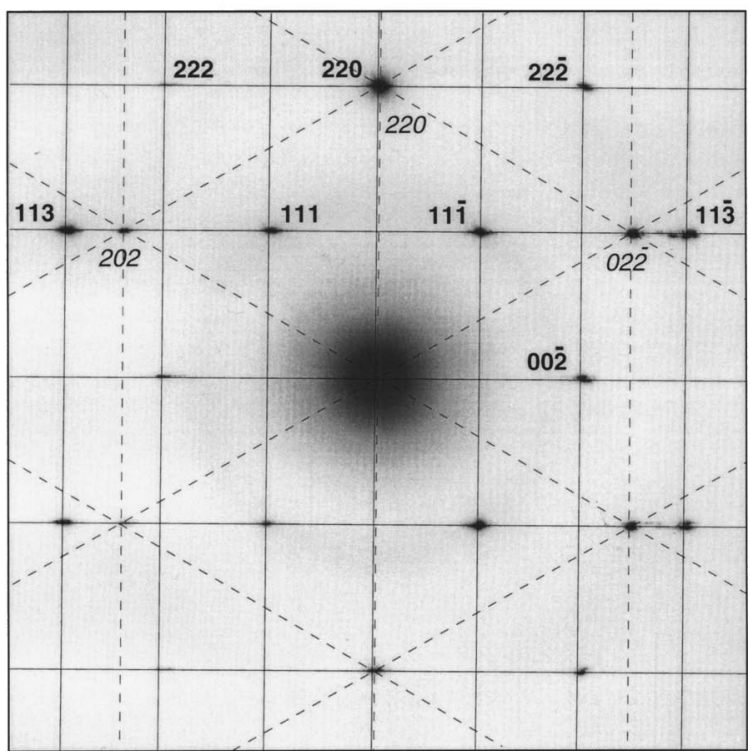

(b)

FIG. 9. Magnified image of electron diffraction from Figs. 3(a) and 4(a). (a) $0^{\circ}$ and $\pm 36^{\circ}$ orientations. Dashed line: [-112] and [1-12] directions with italic characters of Millers' indices scattered from $T 3$ and $T 4$ for $0^{\circ}, T 1$ and $T 5$ for $+36^{\circ}$, and $T 1$ and $T 2$ for $-36^{\circ}$. The indices are only given for the subunit $T 3$ in a $0^{\circ}$ tilt. Full line: [001] direction scattered from $T 1$ for $0^{\circ}, T 3$ for $+36^{\circ}$, and $T 4$ for $-36^{\circ}$. The indices are only given for $T 1$. The (220) reflections are created from all five subunits for all tilts. (b) $\pm 18^{\circ}$. Dashed line: two directions [1-1-1] and [-11-1] from $T 4$ and $T 5$ for positive tilt and from $T 2$ and $T 3$ for negative tilt. Full line: direction [1-10] from $T 2$ and $T 5$ for positive and negative tilts, respectively. The (220) reflections are created for both tilts from all five subunits.

different orientations. For $+36^{\circ}$; these are from $T 5$ in the [1-1-2] orientation, from $T 1$ in the [-11-2] orientation, and from $T 3$ in the [00-1] orientation, respectively. For $-36^{\circ}$, they are from $T 1$ in the [1-1-2] orientation, from $T 2$ in the [-11-2] orientation and from $T 4$ in the [00-1] orientation, respectively. $\pm 18^{\circ}$ tilt: As mentioned before, the PS for the tilts $\pm 18^{\circ}$ resemble the pattern for an [110] zone axis image for the fcc structure. In Fig. 8 only the computer simulation of $+18^{\circ}$ tilt is shown. As explained above, it can be seen from Fig. 7(b) that only half of the image shows atomic resolution. The reflections labeled $1, \ldots, 3$ in Fig. 4 can be explained as follows: Reflection 3 is the (002) reflection, and reflections 1 and 2 are the (11-1) and (111) reflections created by $T 2$. In the diffraction image shown in larger magnification in Fig. 9(b), more reflections are visible. The indices are given for the case of a $+18^{\circ}$ tilt. Three orientations of different subunits are again equivalent to the fcc structure with distortions. The dashed lattice with italic letters for the indices are in the [1-1-1] and [-11-1] orientations for $T 4$ and $T 5$, and in the [1-10] orientation for the lattice marked with full lines. The latter orientation applies for subunit $T 2$ in the case of $+18^{\circ}$ tilt. The (220) reflection is caused by all five subunits. The scattering for $-18^{\circ}$ tilt can be explained in a similar way. However, the scattering subunits are $T 2, T 3$, and $T 5$, respectively. Again all five subunits cause a (220) reflection.

As can be understood from diffraction patterns in larger magnification shown in Figs. 9(a) and 9(b) reflections caused by multiple effects can be identified. All reflections which are not positioned on the two lattices in reciprocal space are caused by multiple effects. Only the reflections labeled in Fig. 2 are explained here. However, there are more multiplescattering reflections present in the diffraction patterns which can also be explained in a similar way. Reflection 5 can be explained as scattering by an overlap of reflections 1 and 7 . Reflection 6 is caused by the negative of reflection 1 and by reflection 4. Reflection 3 is then produced as a multiplescattering effect by an overlap of reflection 5 and the negative of reflection 6 . Reflection 2 is caused by multiple scattering of reflections 1 and -3 . Reflection 8 is a multiple scattering from reflections -1 and 6 .

We also checked the structures of icosahedral particles with additional intermediate planes stapled along one fivefold axis. Icosahedra are also possible structures for small spherical-shaped $\mathrm{Cu}$ clusters. ${ }^{17-19}$ This model, however, was not in agreement with the experiments and was therefore, excluded.

The structure of copper rods as prepared according to the presentation above can be explained clearly as truncated large decahedra. Obviously, due to the additional surfactant in the present preparation at the decahedral nuclei, the growth is hindered and can only take place in the [110] direction, i.e., the fivefold axis, resulting in well-defined long rods.

\section{ACKNOWLEDGMENTS}

This work was supported by the program PROCOPE 1998, "Structural Investigations of Copper Nanoparticles of Different Size and Morphology." The authors thank the French Foreign Ministry (Ministère des Affaires EtrangèresMAE) and the DAAD (Deutscher Akademischer Austauschdienst) for financial support. 
*Author to whom correspondence should be addressed.

${ }^{1}$ M. P. Pileni, Langmuir 13, 3266 (1997).

${ }^{2}$ M. D. Glossman, M. P. Iniguez, and J. A. Alfonso, Z. Phys. D 22, 541 (1992).

${ }^{3}$ I. Lisiecki and M. P. Pileni, J. Am. Chem. Soc. 115, 3887 (1993).

${ }^{4}$ I. Lisiecki, F. Billoudet, and M. P. Pileni, J. Phys. Chem. 100, 4160 (1996).

${ }^{5}$ C. Petit, P. Lixon, and M. P. Pileni, J. Phys. Chem. 97, 12974 (1993).

${ }^{6}$ I. Lisiecki and P. Pileni, J. Phys. Chem. 99, 5077 (1995).

${ }^{7}$ A. S. Bommannavan, P. A. Montano, and M. J. Yacaman, Surf. Sci. 156, 426 (1985).

${ }^{8}$ M. P. Pileni, J. Phys. Chem. 97, 6961 (1993).

${ }^{9}$ M. P. Pileni, B. W. Ninham, T. Gulik, J. Tanori, I. Lisiecki, and A. Filankembo, Adv. Mater. 11, 1358 (1999).

${ }^{10}$ J. Tanori and M. P. Pileni, Adv. Mater. 7, 862 (1995).

${ }^{11}$ J. Tanori and M. P. Pileni, Langmuir 13, 639 (1997).

${ }^{12}$ M. P. Pileni, T. Gulik, J. Tanori, A. Filankembo, and J. C. Dedieu, Langmuir 22, 7359 (1998).

${ }^{13}$ J. Urban, Cryst. Res. Technol. 33, 1009 (1998).

${ }^{14}$ D. Reinhard, P. Berthoud, D. Ugarte, B. D. Hall, and R. Monot, in Proceedings of the Second International Conference on Atomic and Nuclear Clusters, Santorini, Greece, 1993, edited by G. S. Anagnostatos and W. von Oertzen (Springer-Verlag, Berlin, 1995), p. 120.
${ }^{15}$ R. Hillebrand, H. Hofmeister, K. Scheerschmidt, and J. Heydenreich, Ultramicroscopy 49, 252 (1993).

${ }^{16}$ K. Ogawa and S. Kajiwora, Mater. Trans, JIM 34, 1169 (1993).

${ }^{17}$ J. Urban, H. Sack-Kongehl, and K. Weiss, Z. Phys. D 36, 73 (1996).

${ }^{18}$ J. Urban, H. Sack-Kongehl, and K. Weiss, High Temp. Mater. Sci. 36, 155 (1996).

${ }^{19}$ J. Urban, H. Sack-Kongehl, and K. Weiss, Catal. Lett. 49, 101 (1997).

${ }^{20}$ G. G. Granqvist and S. Buhrmann, J. Appl. Phys. 47, 2200 (1976).

${ }^{21}$ F. Frank, W. Schulze, B. Tesche, J. Urban, and B. Winter, Surf. Sci. 156, 90 (1985).

${ }^{22}$ J. M. Cowley and A. F. Moodie, Acta Crystallogr. 10, 609 (1957).

${ }^{23}$ J. Urban, H. Sack-Kongehl, and K. Weiss, Z. Phys. D 28, 247 (1993).

${ }^{24}$ S. Valkealahti and M. Manninen, Phys. Rev. B 45, 9459 (1992).

${ }^{25}$ C. Mottet, G. Tréglia, and B. Legrand, Surf. Sci. 383, L719 (1997).

${ }^{26}$ D. Reinhard, P. Berthoud, D. Ugarte, B. D. Hall, and R. Monot, in Proceedings on Atomic and Nuclear Clusters, Santorini, Greece, edited by G. S. Anagnostatos and W. von Oertzen (Springer-Verlag, Berlin, 1993), p. 128.

${ }^{27}$ J. Urban, Cryst. Res. Technol. 33, 1009 (1998). 